\title{
Refining the treatment of spinal cord lesions: experience from 500 cases
}

\author{
*Manfred Westphal, MD, Klaus C. Mende, MD, and Sven O. Eicker, MD \\ Department of Neurosurgery, University Medical Center Hamburg-Eppendorf, Hamburg, Germany
}

OBJECTIVE Tumorous lesions of the spinal cord, as well as some vascular lesions like cavernous hemangiomas, demand careful consideration as to the indication and approach for surgery. As these lesions are rare in any departmental series, refinement of treatment strategies evolves over long periods. In this context, the authors evaluated a series of 500 intramedullary lesions for approach, technique, outcome, complications, and follow-up.

METHODS Five hundred intramedullary lesions in 460 patients were treated with a continuously evolving departmental strategy between 1985 and 2020. No lesions of the cauda equina or filum terminale were included. The focus of the evaluation was on the adaptation of exposure, resective methodology, sequelae, imaging, and rate of recurrence. Thirtyseven patients were children at the time of diagnosis.

RESULTS Among the 348 neoplastic lesions, the largest subtype was ependymoma ( $n=192,55.2 \%$ ), followed by astrocytoma $(n=89,25.6 \%)$. As a trend, metastases $(n=21)$ have become more frequent and more apparent only in the past 15 years. Reoperations for recurrent or progressive cases or referrals after incomplete resection were performed in 56 cases, mostly for progressive diffuse or pilocytic astrocytomas. Among the vascular lesions, 68 (54.8\%) were hemangioblastomas, followed by $56(45.2 \%)$ cavernous hemangiomas. All intramedullary tumors were approached through a midline myelotomy, refining an en bloc resection technique for endophytic tumors to increase the rate of radical resection. Cavernous hemangiomas reaching the surface and hemangioblastomas were approached directly. Complete removal was possible in $77.2 \%$ of endophytic tumors but in only $41.7 \%$ of diffuse tumors. All WHO grade II diffuse astrocytomas, WHO grade III tumors, and glioblastoma progressed despite treatment according to standard regimens. Vascular lesions were regularly removed completely, with only 1 recurrence of a large hemorrhagic thoracic cavernous hemangioma. The major sequelae were sensory deficits and neuropathic pain. Stabilizing instrumentation was placed in 5 cases of spinal deformity, mostly when more than 4 levels were affected, and in the pediatric population.

CONCLUSIONS In a large series of intramedullary surgeries, refinement of treatment strategies related to exposure, implementation of intraoperative adjuncts such as ultrasound, intraoperative neuromonitoring, resective strategies, and reconstruction were evaluated. The authors found that for almost any defined, endophytic medullary lesion, a safe and complete removal can be offered.

https://thejns.org/doi/abs/10.3171/2021.2.FOCUS201107

KEYWORDS spinal cord lesion; intramedullary; surgery; cavernoma; angioblastoma; ependymoma; astrocytoma

I NTRINSIC tumors of the spinal cord represent $2 \%$ to $10 \%$ of all neoplasms of the $\mathrm{CNS},{ }^{1-7}$ in reality being at the lower end of the range. They are more common in young adults ${ }^{4}$ and have a different histological spectrum than in the adult group. Surgery of these complex and rare lesions is challenging, as the cord is a densely functional and vulnerable structure. Evolving microsurgical strategies, expanding imaging technologies, and a growing number of technological adjuncts have led to constant evolution and refinement of spinal cord surgery, which we revisited here in a large institutional series that focuses on space-occupying intramedullary lesions, including intrinsic or metastatic tumors, but also hemangioblastomas (HAs) and cavernous hemangiomas.

Because intramedullary spinal cord lesions (IMSCLs) are uncommon and often present with subtle symptoms, diagnosis is often delayed $; 8$ the primary counseling about the nature of the lesion and presumably dismal prognosis often generate unwarranted anxiety in patients. Treatment inevitably involves surgery, in most cases for complete or maximally safe resection, but also for biopsy. Biopsy should be limited to select cases, as the findings oftentimes

ABBREVIATIONS CEG = Cooper-Epstein grade; $\mathrm{HA}=$ hemangioblastoma; $\mathrm{IMSCL}=$ intramedullary spinal cord lesion; $I O N M=$ intraoperative neuromonitoring; $\mathrm{MPS}=$ methylprednisolone; NASCIS II = National Acute Spinal Cord Injury Study 2; MEP = motor evoked potential; SSEP = somatosensory evoked potential.

SUBMITTED January 1, 2021. ACCEPTED February 17, 2021.

INCLUDE WHEN CITING DOI: 10.3171/2021.2.FOCUS201107.

* M.W. and K.C.M. contributed equally to this work. 
TABLE 1. Spectrum of spinal cord lesions in adults and infants from 1985 to 2020

\begin{tabular}{lrrr}
\hline & \multicolumn{3}{c}{ No. of Lesions (\%) } \\
\cline { 2 - 4 } & In Adults & In Children & Total \\
\hline Ependymoma & $184(36.8)$ & $8(1.6)$ & $192(38.4)$ \\
\hline Astrocytoma & $70(14.0)$ & $19(3.8)$ & $89(17.8)$ \\
\hline Glioblastoma & $6(1.2)$ & $2(0.4)$ & $8(1.6)$ \\
\hline Oligodendroglioma & $4(0.8)$ & $0(0.0)$ & $4(0.8)$ \\
\hline Melanocytoma & $4(0.8)$ & $0(0.0)$ & $4(0.8)$ \\
\hline Ganglioglioma/-cytoma & $9(1.8)$ & $4(0.8)$ & $13(2.6)$ \\
\hline Lipoma & $12(2.4)$ & $1(0.2)$ & $13(2.6)$ \\
\hline Metastasis & $21(4.2)$ & $0(0.0)$ & $7(1.4)$ \\
\hline Inflammatory & $6(1.2)$ & $1(0.2)$ & $10(2.0)$ \\
\hline Cystic lesion & $9(1.8)$ & $1(0.2)$ & $4(0.8)$ \\
\hline Teratoma & $1(0.2)$ & $3(0.6)$ & $56(11.2)$ \\
\hline Inconclusive biopsy & $10(2.0)$ & $1(0.2)$ & $68(13.6)$ \\
\hline Cavernoma & $53(10.6)$ & $3(0.6)$ & $500(100)$ \\
\hline Angioblastoma & $66(13.2)$ & $2(0.4)$ & \\
\hline Total & $455(91.0)$ & $45(9.0)$ & \\
\hline
\end{tabular}

are inconclusive; the procedure should be reserved for a priori unresectable cases or histological confirmation for implementation of other treatment modalities, ${ }^{9}$ especially in cases of lymphoma. ${ }^{2,4}$ Notwithstanding the poor expectations, advancements in and defined limits of the surgical procedures allow for safe treatment of most lesions. There are numerous reports containing rather small series..$^{10-14}$ Acknowledging that different but equivalent approaches may evolve in different centers, we present our series of 500 cases not in an attempt to prescribe definitive standards, but to contribute to the advancements in intramedullary lesion surgery in general. In this study, a variety of sizable defined subgroup lesions were treated surgically in the same center, with techniques and strategies evolving as the series grew and as constant adaptations were made from earlier reports. ${ }^{15-17}$

\section{Methods}

Records from 500 surgeries in 460 patients for IMSCLs were either retrieved from archives or documented in a prospective database. Histology was based on the respective WHO classification used at the time, and no reevaluation was performed according to the new WHO 2016 classification for cases from earlier decades (Table 1). Symptoms and neurological deficits were scored using the Cooper-Epstein grade (CEG), ${ }^{18}$ which allowed for a standardized evaluation of clinical change in the perioperative and follow-up periods.

All patients underwent Gd-enhanced whole-spine MRI at the time of diagnosis to assess possible multiple lesions and enable level counting from below. The extent of resection was based on Gd-enhanced postoperative MRI performed within 6 weeks postoperatively at the latest and preferably within 48 hours for contrast-enhancing lesions. Total intravenous anesthesia was routinely used, and an adequate perfusion pressure (a maximum of a mean ar- terial blood pressure of approximately $80 \mathrm{~mm} \mathrm{Hg}$ ) was maintained. Intraoperative neuromonitoring (IONM) is a standard of spinal cord surgery, and motor evoked potential (MEP), somatosensory evoked potential (SSEP), and $\mathrm{D}$-wave monitoring have routinely been employed during tumor resection since 1996. A methylprednisolone (MPS) regimen based on the National Acute Spinal Cord Injury Study 2 (NASCIS II) findings for traumatic spinal cord injuries was initiated before the dura mater was opened.

Data analysis was performed using IBM SPSS Statistics version 26 (IBM Corp.) and Microsoft Excel 2019. Cross tables and chi-square test analyses were performed for nominal and ordinal data. Parametric data were analyzed using the Student t-test. A logistic regression analysis was performed for the impact of preoperative clinical status on clinical outcome. All results were deemed significant at $\mathrm{p}<0.05$.

\section{Results}

Between 1985 and 2020, 500 intramedullary lesions were surgically treated. Of these lesions, 348 were tumors, with ependymomas being the most prominent $(n=192$; Fig. 1), followed by astrocytomas of all grades $(n=89$; Fig. 2). There were 124 vascular pathologies (Fig. 3). The remaining 28 pathologies were intramedullary cysts $(\mathrm{n}=$ $10)$, diffuse inflammation $(n=7)$, or had no conclusive pathology $(\mathrm{n}=11$; Fig. 4 , Table 1$)$. The mean patient age was $41 \pm 17$ years, with statistically significant differences between subgroups and growth patterns: endophytic, $44 \pm 15$ years; diffuse, $34 \pm 18$ years; vascular, $41 \pm 14$ years; and metastases, $54 \pm 19$ years (ANOVA, $\mathrm{p}<0.001$; differences between diffuse and all other groups were significant at $\mathrm{p}$ $<0.01$ [Bonferroni], as was the difference between vascular and metastatic lesions). The mean time from initial symptoms to diagnosis was $93 \pm 114$ weeks, which was not significantly different between the subgroups. 

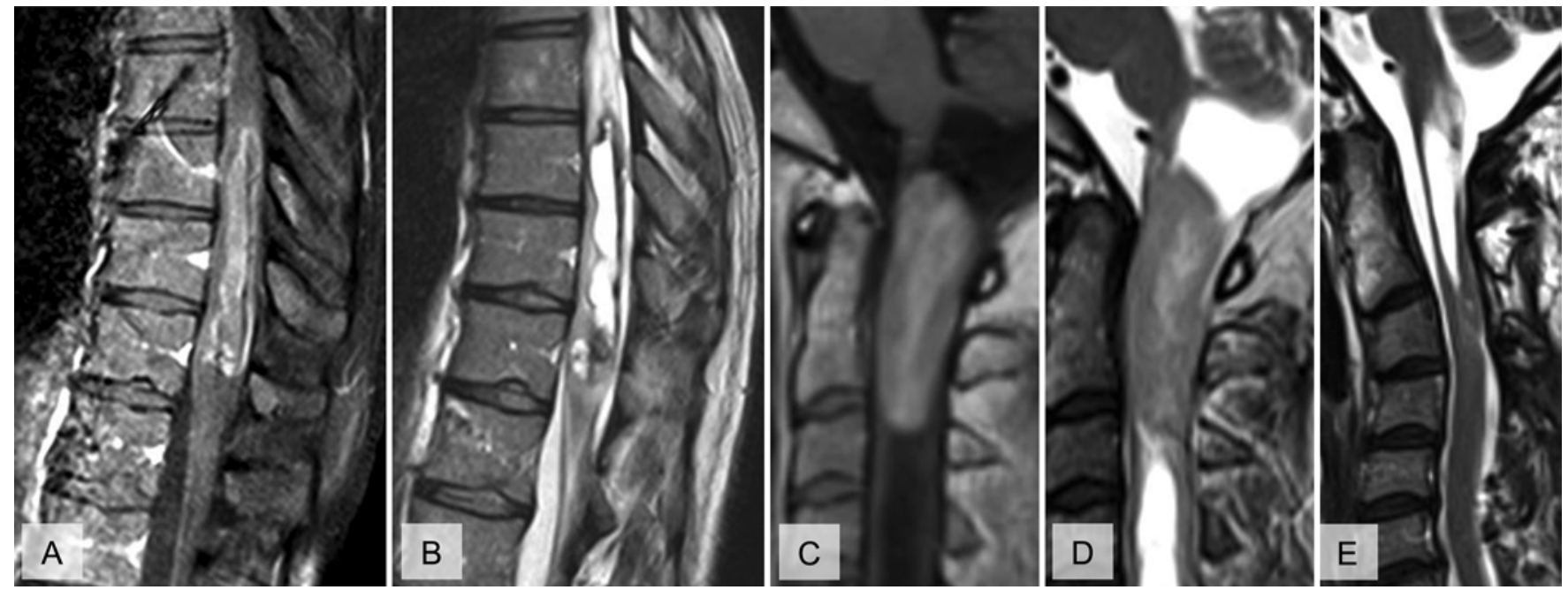

FIG. 1. Sagittal MR images of representative spinal cord ependymomas. A: Contrast-enhanced T1-weighted image showing the intramedullary tumor from T10 to T12. B: Image showing different parts with partially solid and cystic proportions. C: Contrast-enhanced T1-weighted image showing a second case with extended spinal cord ependymoma from C0 to C3. D: Image demonstrating the corresponding syringomyelia. E: Image showing the postoperative result after complete tumor removal.

\section{Clinical Status}

In 19\% (74/390) of patients, the preoperative CEG was 0 for both upper and lower extremities, and the lesions were discovered incidentally. Of the remaining patients, $58.5 \%$ presented with very mild deficits (CEG $0-1), 25.6 \%$ with mild deficits (CEG 2), and $11.5 \%$ with moderate to high deficits (CEG 3); $4.4 \%$ presented with major impairment (CEG 4 or 5) and severe deficits concerning lower- or upper-extremity function (chi-square test, $\mathrm{p}<0.001$ ). Deficits progressed in $84.0 \%$ of endophytic lesions, $87.7 \%$ of diffuse lesions, $86.4 \%$ of vascular lesions, and $92.3 \%$ of metastatic lesions.

At discharge, upper- and lower-extremity CEGs were improved or equal to those at the first presentation in $69.5 \%$ and $60.6 \%$ of patients, respectively (chi-square test, $\mathrm{p}<0.001)$. On short-term follow-up (6 months), in $40.2 \%$
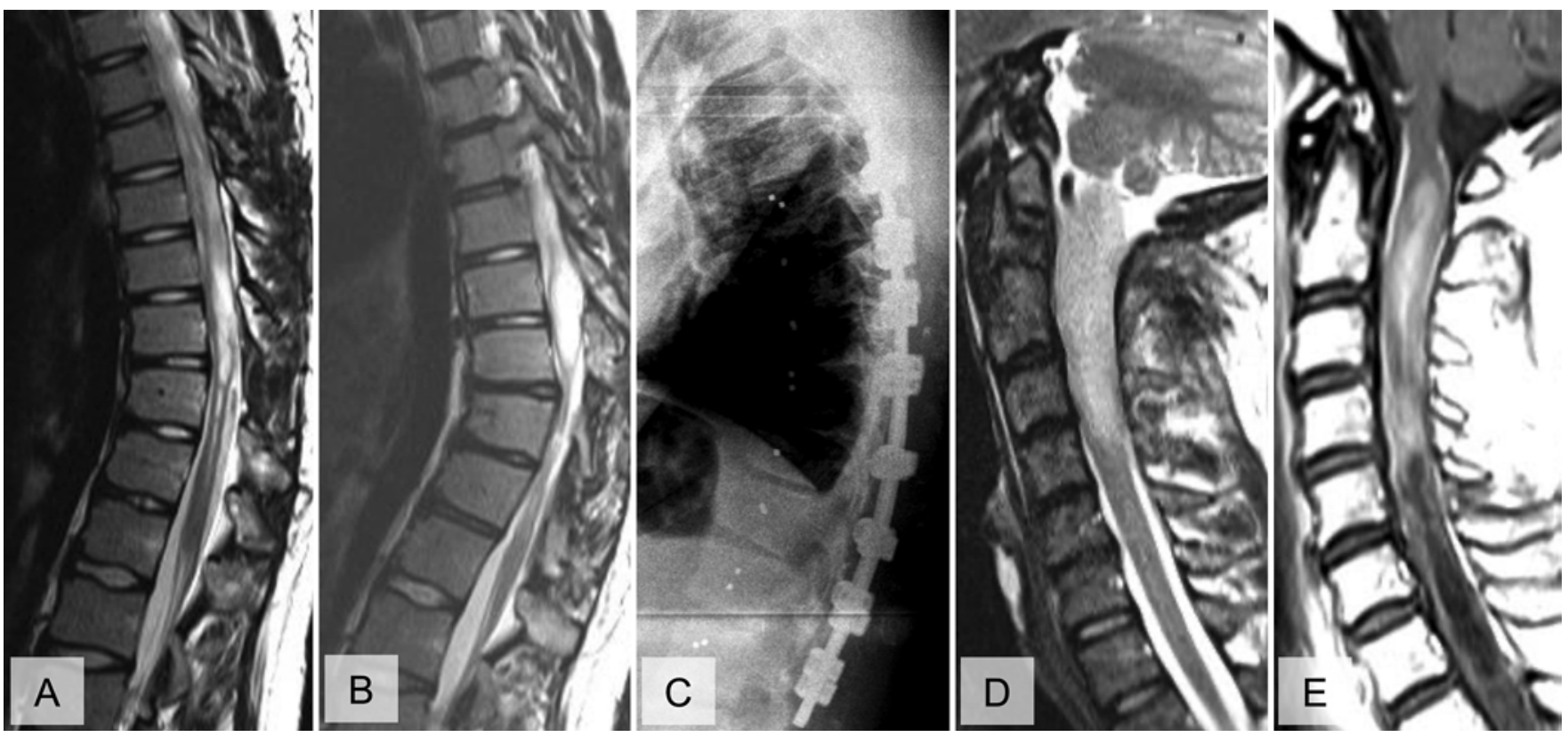

FIG. 2. Exemplary presentation of a pilocytic astrocytoma. A: Sagittal T2-weighted image of the thoracolumbar spine. B and C: Postoperative kyphotic deformity despite reconstruction of the dorsal elements of the spinal column (B) requiring spinal stabilization (C; with carbon fiber-reinforced PEEK screws to reduce susceptibility artifacts on postoperative imaging). D: Sagittal T2-weighted MR image of the cervical spine, showing diffuse astrocytoma (IDH mutated). E: Sagittal T1-weighted MR image of the cervical spine showing anaplastic astrocytoma. 

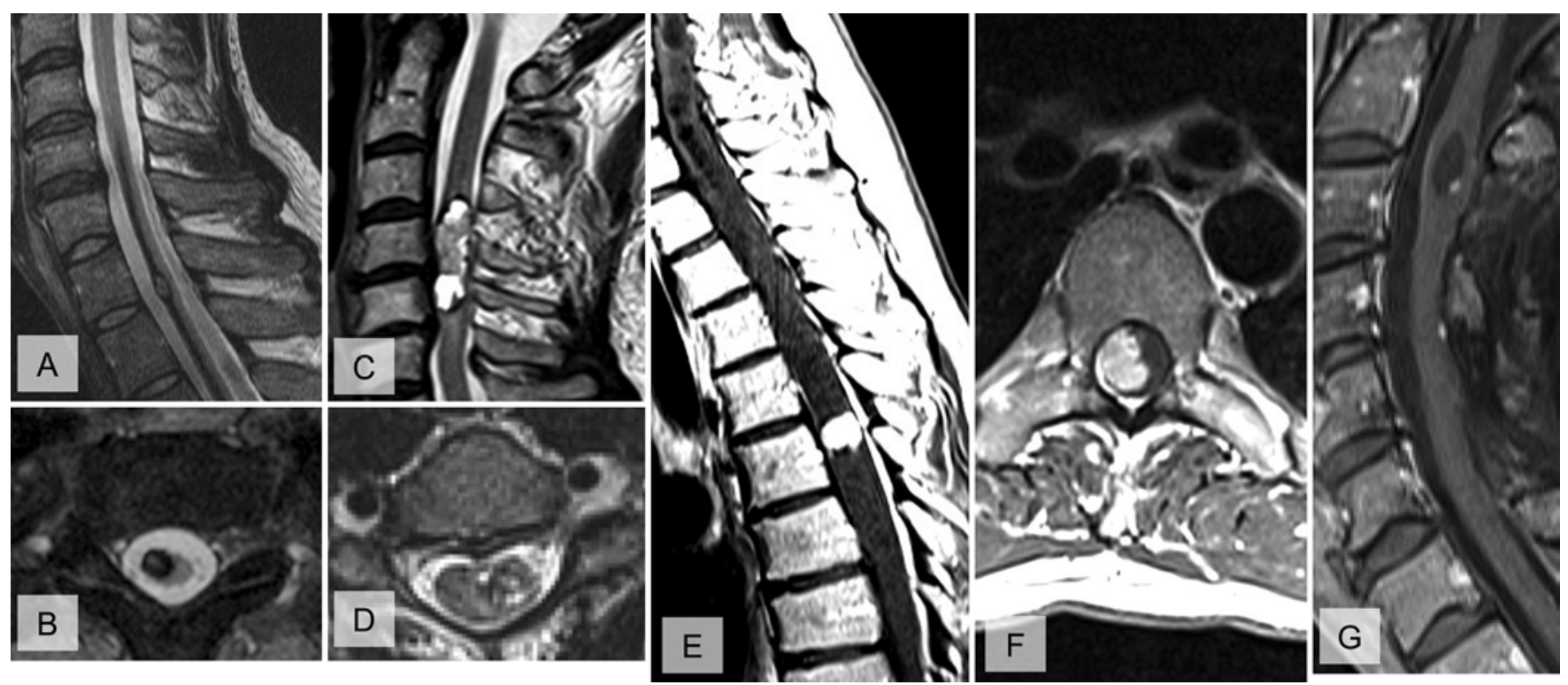

FIG. 3. Vascular lesions. A and B: Sagittal (A) and axial (B) T2-weighted images showing a small eccentrically located cavernous hemangioma. Hemosiderin (black) is clearly visible in panel B. C and D: Sagittal (C) and axial (D) T2-weighted MR images illustrating the dimensions that a cavernous hemangioma can achieve in the cervical spine. E and F: Sagittal (E) and axial (F) images showing a highly vascularized hemangioblastoma of the thoracic spinal cord. G: Sagittal image of a small angioblastoma with a corresponding cyst in the cervical spine.

of patients upper-extremity neurological function further increased, as it did in $24 \%$ for the lower extremities. In total, stabilization or improvement at the 6-month follow-up was recorded in $88.4 \%$ of patients for the upper extremi- ties (Table 2) and $58.5 \%$ of patients for the lower extremities (Table 3).

Improvement in lower-extremity function at 6 months after surgery was more likely if the preoperative neuro-

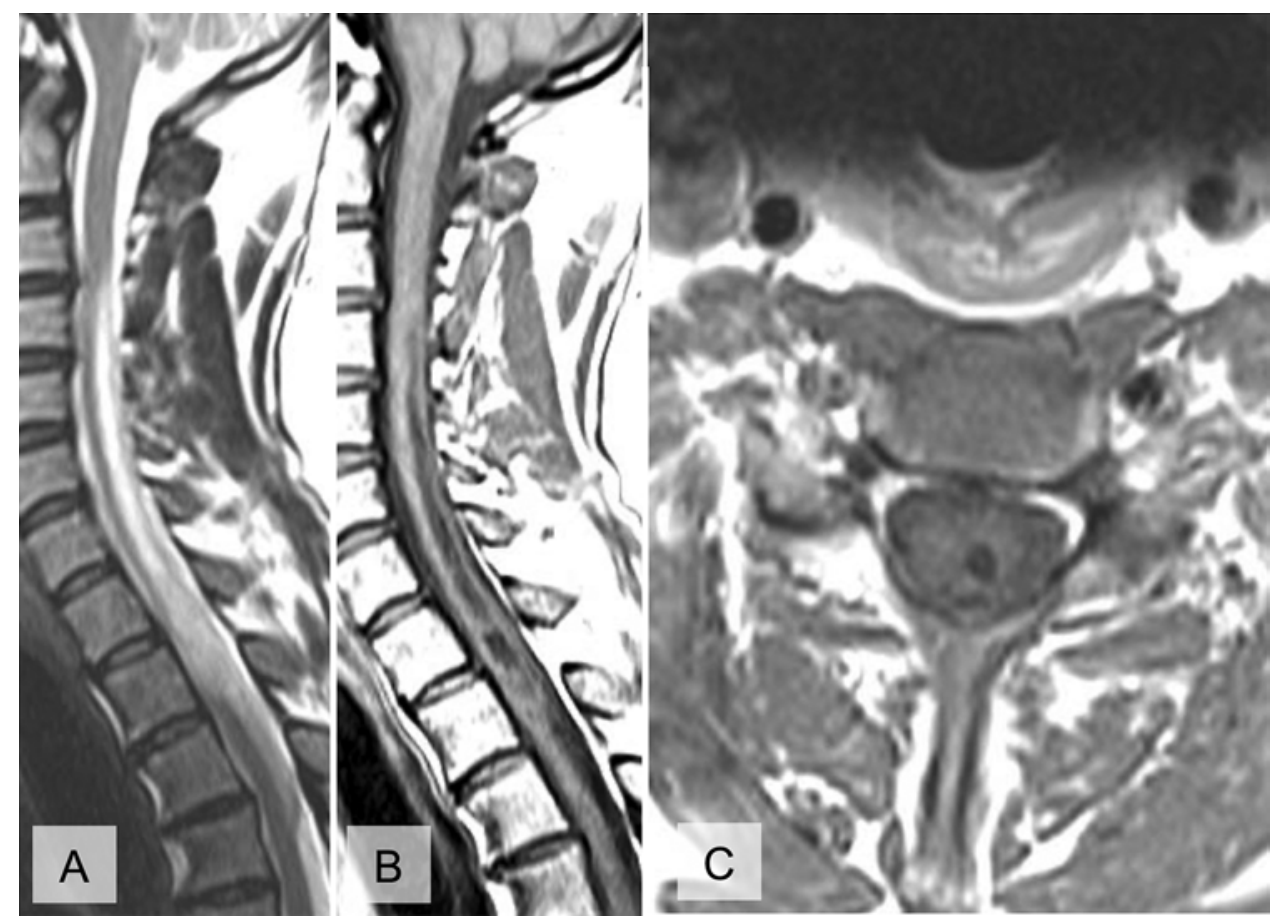

FIG. 4. A and B: Sagittal T2- (A) and T1-weighted (B) MR images of the cervical spine, showing a rare case of unclear extended neoplastic tethering of the spinal cord, with cystic and solid tumor parts and corresponding syringomyelia. C: Axial image of the T2 vertebra. The etiology remains unclear even after biopsy. 
TABLE 2. Lower-extremity CEGs at 6 months

\begin{tabular}{|c|c|c|c|c|c|c|c|}
\hline \multirow[b]{2}{*}{ Postop CEG } & \multicolumn{6}{|c|}{ Preop CEG } & \multirow[b]{2}{*}{ Overal } \\
\hline & 0 & 1 & 2 & 3 & 4 & 5 & \\
\hline 0 & $8.0 \%$ & $11.5 \%$ & $4.0 \%$ & $1.5 \%$ & $1.5 \%$ & $0.0 \%$ & $26.5 \%$ \\
\hline 1 & $3.0 \%$ & $23.0 \%$ & $8.5 \%$ & $1.0 \%$ & $1.5 \%$ & $0.0 \%$ & $37.0 \%$ \\
\hline 2 & $0.0 \%$ & $3.5 \%$ & $10.0 \%$ & $7.0 \%$ & $1.0 \%$ & $0.0 \%$ & $21.5 \%$ \\
\hline 3 & $0.5 \%$ & $0.5 \%$ & $2.5 \%$ & $4.5 \%$ & $2.5 \%$ & $1.0 \%$ & $11.5 \%$ \\
\hline 4 & $0.0 \%$ & $0.5 \%$ & $0.5 \%$ & $1.0 \%$ & $1.0 \%$ & $0.5 \%$ & $3.5 \%$ \\
\hline 5 & $0.0 \%$ & $0.0 \%$ & $0.0 \%$ & $0.0 \%$ & $0.0 \%$ & $0.0 \%$ & $0.0 \%$ \\
\hline Overall & $11.5 \%$ & $39.0 \%$ & $25.5 \%$ & $15.0 \%$ & $7.5 \%$ & $1.5 \%$ & \\
\hline
\end{tabular}

The values across the diagonal (shown in shading and/or bold) represent the stable patients. The values under the diagonal represent patients who declined, and the values above the diagonal represent patients who experienced neurological improvement. Improvement was seen in $12 \%, 46.5 \%$ remained stable, and $41.5 \%$ had a decline.

logical impairment was minor (OR 1.704/CEG for a stable or better outcome, 95\% CI 1.27-2.29; logistic regression, $\mathrm{p}<0.001)$.

\section{Recurrent Lesions}

Fifty-six patients in our cohort had recurrent lesions. Of all endophytic tumors, 21 were surgeries for recurrent tumor (15 were previous operations at our institution and 6 were external referrals). In diffuse tumors, 26 were recurrent lesions (19 of these were previous operations at our institutions and 7 were external), and for metastases, only 1 recurrent case was treated. Eight surgeries were performed on a recurring vascular lesion. Because of the technical advances made over the course of this study, recurrence rates were significantly reduced over time; patients operated on before the year 2000 had an overall recurrence rate of $19.8 \%$ versus $7.2 \%$ for patients operated on after that year (chi-square test, $\mathrm{p}=0.001$ ).

\section{Surgery}

All intrinsic tumors are approached through the midline, so the exposure is by laminotomy. Care is taken to gain adequate access to the poles of the tumors, so that there is a straight view to the entrance of the ventral dissection plane. After exposure of the dura mater, adequate exposure is verified by ultrasound so that the edges of the lamina above or below can be removed if necessary while the dura mater is still closed (Fig. 5). In cases in which the arachnoid space is obliterated and the dura mater is opened, the cord may rise out of the opening. To avoid injury in such cases of swelling, it is mandatory to drain cerebrospinal fluid from the ventral space above the lesion.

The main aspect of this series was the use of a $\mathrm{CO}_{2}$ laser in an attempt at in toto removal of endophytic tumors, as illustrated in Fig. 6B and C. By dislodging the tumor from its bed, thereby alternating from pole to pole, severing adhesions with $1.5 \mathrm{~W}$ of $\mathrm{CO}_{2}$ laser power, and uplifting the increasingly mobile tumor, the dissection plane is opened with minimal traction. This allows for stripping the tracts from the tumor surface, resulting in radical removal. With clear visibility of the ventral vessels entering and branching at the surface of the tumor, avoidance of injury to the anterior spinal artery was possible in all cases.

In diffuse tumors, in which the midline approach does not open a clear dorsal tumor surface and dissection plane on which the tumor can be followed in its circumference (Fig. 6), color and texture may allow for an internal decompression and decreasing intramedullary pressure throughout debulking. This results in the appearance of a transition zone that may be carefully "thinned out," considering that fiber tracts will be embedded in the outer layer. For pilocytic astrocytoma or diffuse astrocytoma grade II, this may still lead to long-term control. Glioblastoma and metastases are also only debulked inside the margins of change of

TABLE 3. Upper-extremity CEGs at 6 months

\begin{tabular}{ccccccc}
\hline & \multicolumn{7}{c}{ Preop CEG } & \multicolumn{1}{c}{ Overall } \\
\cline { 2 - 6 } Postop CEG & \multicolumn{1}{c}{1} & 2 & 3 & 4 & $47.7 \%$ \\
\hline 0 & $41.7 \%$ & $3.5 \%$ & $2.5 \%$ & $0.0 \%$ & $0.0 \%$ & $30.2 \%$ \\
\hline 1 & $11.1 \%$ & $15.6 \%$ & $3.0 \%$ & $0.5 \%$ & $0.0 \%$ & $18.0 \%$ \\
\hline 3 & $4.0 \%$ & $7.0 \%$ & $5.0 \%$ & $2.0 \%$ & $0.0 \%$ & $3.0 \%$ \\
\hline 4 & $0.5 \%$ & $0.5 \%$ & $1.0 \%$ & $1.0 \%$ & $0.0 \%$ & $1.0 \%$ \\
\hline Overall & $0.0 \%$ & $0.0 \%$ & $0.5 \%$ & $0.5 \%$ & $0.0 \%$ & \\
\hline
\end{tabular}

The values across the diagonal (shown in shading and/or bold) represent the stable patients. The values under the diagonal represent patients who declined, and the values above the diagonal represent patients who experienced neurological improvement. Improvement was seen in $25.1 \%, 63.3 \%$ remained stable, and $11.5 \%$ experienced decline. 


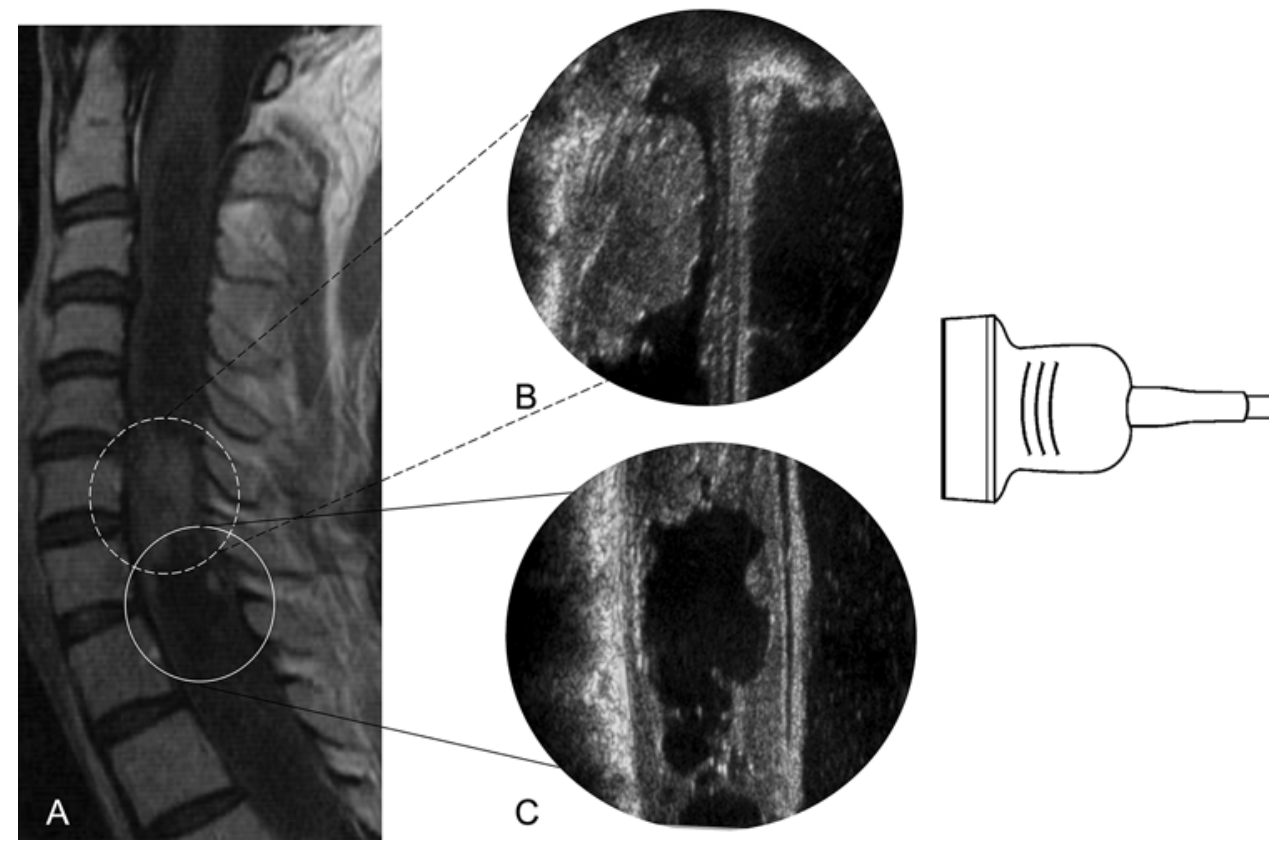

FIG. 5. Intraoperative ultrasound verification of the tumor extent and localization before opening the dura mater. A: Sagittal contrast-enhanced T1-weighted MR image from C1 to T3 with an ependymoma at C5-7. A solid tumor is in the dashed circle (cranial syringomyelia is also displayed). The solid circle shows the tumor cyst. B: The solid tumor is perfectly visualized before opening the dura mater. C: The cystic part of the tumor is shown.

texture quality, followed by standard radiation therapy and chemotherapy. With metastases becoming notably more frequent in the past years and with extended survival of cancer patients, the need for interdisciplinary consultation for chemotherapeutic and especially for radiosurgical options, and adequate sequencing, is emerging..$^{19}$

Gross-total resection was possible in $77.2 \%$ (95/123) of our endophytic tumor cases, but only in $41.7 \%$ (30/72) of the diffuse tumors and $72.7 \%$ of the metastases (chi-square test, $\mathrm{p}<0.001)$. The $\mathrm{CO}_{2}$ laser was used in $92.9 \%$ of all ependymoma resections. A macroscopically complete resection was achieved in $82.0 \%$ of all cases in which a $\mathrm{CO}_{2}$ laser was employed. Consequently, the recurrence rate of ependymomas throughout the whole series, including the "learning curve," was $17.5 \%(28 / 160), 14.0 \%(n=13)$ for those that underwent total resection and $31.8 \%(\mathrm{n}=7)$ for subtotal resection (chi-square test, $\mathrm{p}=0.11$ ).

Vascular lesions such as HA and cavernous hemangioma have clear margins, mostly delineated by a clear plane of gliosis (Fig. 6). Cavernoma can be gradually shrunk by draining its cyst so that it is dissected away from the gliosis into the cavity resulting from evacuation. HAs, on the contrary, are left intact and are carefully devascularized in their pial embedding, arteries before veins, and then after opening the pia circumferentially, slowly shrunk with the bipolar away from the gliotic bed, and finally removed in toto to prevent recurrence. Vascular lesions were totally removed in $93.4 \%$ of cases.

\section{Electrophysiological Findings}

SSEPs were found to frequently decrease by $30 \%$ unilaterally with the midline incision. This was indicative of differential vulnerability due to asymmetrical tumor growth and indicated the side of more adhesiveness or surface assimilation to fiber tracts or invasion. Sudden changes in SSEPs were seen when too much pressure was exerted when the dissection plane was opened on one side. In response, we let that area rest and continued on the contralateral side. Slowly diminishing SSEPs and MEPs were observed when duration of surgery was long and were disregarded when no surgical maneuver could be associated with the decreases. These monitoring changes usually occurred later in the procedure, and the resection was always completed to the extent anticipated for the respective lesion.

\section{Complications and Neurological Sequelae}

CSF leaks requiring surgical intervention were detected in 8 cases. Revision surgery due to postoperative hematoma was necessary for 4 cases. There were no surgical site infections.

As for sensory findings, nearly all patients who had their tumor accessed through a midline approach reported dysesthesia of the lower body and diminished proprioception in the immediate postoperative period; however, these symptoms did not impair their walking if there was no preexisting severe motor deficit. The sensory deficits were always more pronounced on the side where the tumor was more adherent to the inner surface of the tumor cavity reflecting the area of origin, which especially in endophytic astrocytoma may be an area of unilateral infiltration opposite a clean dissection plane contralaterally. A small proportion of patients developed neuropathic pain as gliosis became part of the postoperative consolidation of the resection cavity. 


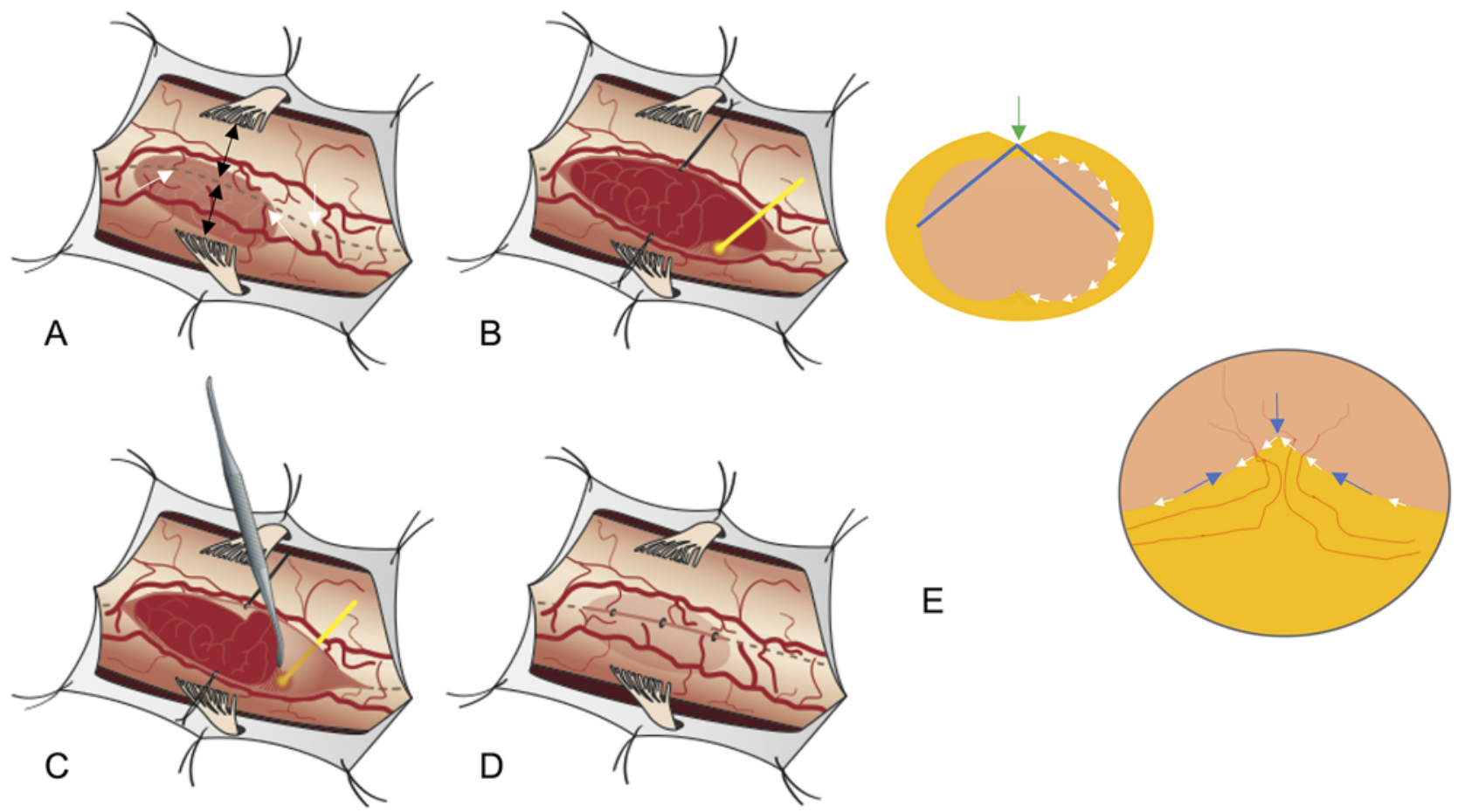

FIG. 6. A: Spinal cord alignment altered by an IMSCL with hypervascularization, midline shift (black arrows), and bulging of the spinal cord. Midline identification can be achieved by perforating vessels (white arrows), nerve root symmetry, and visualization of the dorsal median sulcus (dashed gray line). B: Pial stay sutures (black lines) are advocated by some but are met with skepticism by others. The $\mathrm{CO}_{2}$ laser (yellow line) may be helpful in lateral tumor dissection. C: After lateral and pole dissection, the tumor may be lifted, and the plane of dissection followed with a $\mathrm{CO}_{2}$ laser to cut even small adhesions. D: Using pial sutures to close the cord may prevent complications through adhesions. $\mathrm{E}$ : The midline or dorsal sulcus offers an approach from which the lateral aspects can be reached with near equidistance (blue lines). The direction of dissection along the surface (white arrows), approaching the midline from both sides (blue arrows), and localization of feeding vessels in the ventral part of the spinal cord.

\section{Spinal Deformity}

Laminotomies have been used for exposure in all primary cases of this series since 1990. In recurrent cases in which lesion removal became difficult because of extensive fibrosis, stepwise laminectomy was performed to protect the dural sac. Even with laminotomy, extensive cervical swan neck deformity developed in 4 patients and progressive thoracic kyphosis in 1 patient (Fig. 2A-C); all were children. This developed over a course of 1 to 5 years and led to spinal instrumentation. In adults, mild swan neck deformity that did not cause neurological symptoms progressed in some patients for 3 to 5 years and then stabilized, with patients refusing offered corrections after the deformity became fixed and no surgery was required if there was no recurrence. Spinal deformity was more likely to occur in multilevel laminotomies of 4 or more segments.

\section{Discussion}

We present a large and complex series of institutional experience with intramedullary surgery, mostly for tumors, which is the continuous experience of a very small team (see Acknowledgments) over a specific period. ${ }^{16-21}$ Our main finding was that there are some general principles that should be standardized and followed in every surgery, but there are also entity-specific strategies that need to be adopted.
For exposure of nonexophytic, completely intramedullary tumors, mostly ependymoma and astrocytoma, we exclusively used the midline approach to the cord, as have many others. ${ }^{22-26}$ With the tumor mass mostly located in the central cord, the midline or dorsal sulcus offers an approach from which the lateral aspects can be reached with almost equidistance without unnecessarily injuring fiber tracts (Fig. 6E). Thus, no asymmetrical strain is put on the tumor surroundings as in the dorsolateral approach, which is proposed as an alternative. ${ }^{27}$ Identifying the midline for a longitudinal incision of the pia mater can be difficult due to the altered anatomy and torsion in the presence of an underlying tumor with asymmetrical cord distension (Fig. $6 \mathrm{~A})$, but vessels perforating at the midline into the depth of the cord serve as a landmark, as may the dorsal root entry zones. In contrast, exophytic lesions, often cavernoma and $\mathrm{HA}$, are approached from their exposed surface, and for ventrolateral lesions, a longer exposure and rotation of the cord by detaching and gently pulling on the dental ligament allows access.

Likewise, the method of tumor removal has to be individually adapted, but we advocate that solid intramedullary tumors should be left intact as described above. This en bloc removal appears to be crucial for obtaining high rates of complete resections for well-circumscribed intramedullary tumors, as is also reported by other authors. ${ }^{28}$ 
By outward traction or pushing of the tumor starting at the poles, the dissection plane to be targeted opens up. Thus, slowly approaching the equator from the cranial as well as the caudal pole in order to raise only half the tumor at the time, the traction enables a safe severance of the fiber tracts surrounding the tumor surface. Working along the clearly visible tumor/cord interface, it has even been claimed that IONM may not be necessary in cases resected with this technique. ${ }^{28}$ This approach may have to be modified in long tumors that extend over 3 or 4 segments, so that a completely freed segment is cut off and a new starting pole is created to continue along the same methodology. In our series, what has greatly facilitated this resective technique is use of the $\mathrm{CO}_{2}$ laser, which is used to open up the dissection plane mostly at the lateral aspects where adhesions and vascular bridges resist the microdissector used to shell the tumor out of its bed. Applying a short $\mathrm{CO}_{2}$ laser burst through which the thermal energy dissipates in the act of evaporation of the adhesive spot on the tumor surface ensures maximal safety for the cord tissue (Fig. 6B and C). To keep a long, multilevel myelotomy and enlarging resection cavity open during the length of the procedure is also a matter of controversy-pial 6-0 stay sutures are advocated by some ${ }^{23}$ but are met with skepticism by others (R. Spetzler, personal communication) - so we tend to use them (modified to $8 \times 0$ ) only in long exposures with wide opening over the exposed tumor in the midline so that they more or less "hold" rather than "pull" the well-detached dorsal columns.

While en bloc resection is a decisive aspect for radical removal and has a low recurrence rate for circumscribed tumors, this does not hold true for diffuse or invasive tumors. In these cases, debulking is the first step, possibly opening boundaries at which a transition zone to normal cord tissue becomes apparent. Guidance can be obtained from working with experienced specialists to interpret monitoring or intraoperative stimulation findings, ${ }^{4,29}$ as well as fluorescence in some lesions. ${ }^{4,22}$ However, this is not broadly validated and depends very much on histology, individual texture characteristics, and, foremost, personal experience. In diffuse astrocytomas, where the complete cord is one homogeneous tumor-infiltrated entity, biopsy is advocated only to obtain proper diagnosis to guide therapeutic decisions. Also, neuroglial tumors like gangliogliomas or gangliocytomas, reflecting the essential matrix of the cord, lack a clearly defined border. This only allows a conservative wedge resection guided by consistency and color, with careful attention to the electrophysiology. During such debulking, the $\mathrm{CO}_{2}$ laser has also been found to be helpful.

Emergency situations are rare for intramedullary lesions. Intratumoral hemorrhages may occur in ependymoma and HA and are the typical presenting sign for cavernous hemangiomas. This is usually accompanied by extensive edema, so that high-dose MPS or dexamethasone is recommended to alleviate symptoms. If the level of paraparesis is not higher than grade II, waiting for several days or even weeks to let the cord recover from the impact results in a much less vulnerable perilesional environment.

There is also the issue of how to deal with tumors that are found incidentally and produce no symptoms. When small and nonreactive, these tumors should just be observed with regular MRI follow-up at yearly intervals unless symptoms develop. Slow growth, changed contrast characteristics, and cyst formation may lead to a discussion of preemptive surgery with the patient. This is relevant in HA. Most of our HA cases were spontaneous and therefore single, but patients who have von Hippel-Lindau syndrome frequently present with multiple intramedullary HAs. One study proposed early spinal surgery in these cases, even for asymptomatic lesions, since symptoms, once present, were often found to be irreversible $(71.4 \%$ did not improve after surgery). ${ }^{30}$ We propose a more conservative approach, as there has to be a trade-off between the preventive aspect and the acceptable side effects, which can be disproportionate for small, completely asymptomatic lesions. Since it is hard to predict future developments in patients' lives, leaving as much of the spine intact as possible is our foremost concern.

As for sequelae, spinal deformity may have severe reconstructive consequences. Undisputedly, optimal exposure for a centrally located intramedullary lesion should allow the entire circumference of the tumor to be reached at the equator, with equal bilateral exposure, which is best achieved through a midline approach. Thus, the generally favored keyhole approaches ${ }^{31}$ are inadequate in this situation, except for lateral surface lesions like cavernomas. Multilevel laminotomies in different techniques are now standard; laminectomy has become inappropriate because of frequently observed deformities, especially in the cervical spine and in young patients, causing a paradigm shift in the 1990s..$^{25}$ In a long-term cohort of 55 pediatric patients, new-onset spinal deformity after IMSCL surgery was reported to be as high as $16 \%$, with stabilization required in $55 \%$ of patients. ${ }^{32}$ The risk for deformity is reduced with smaller exposures, ${ }^{33}$ and even further with laminoplasty, ${ }^{34,35}$ but it is still a risk that cannot be completely avoided and should be discussed with the patient prior to obtaining their consent for surgery (Fig. 2). Laminoplasty also offers advantages should recurrence or progression necessitate another exposure.

While patients usually adapt to proprioceptive or sensory deficits, the development of neuropathic pain, ${ }^{25,36}$ which reaches up to $60 \%$ in some series, ${ }^{22}$ can be a dramatic sequela. This can occur during gliotic scar consolidation of the resection cavity and may fluctuate within a period of 2 years postoperatively, but thereafter it becomes a burden to the patient as it may even increase over time. Adhesions of the pial opening to the dorsal dura mater incision can result in tethering causing microtrauma and can worsen over time, so untethering may be necessary, but may still recur. Pain management is difficult and may require multimodal interventions. Limiting its occurrence, pial closure of the resection cavity with microsutures is attempted wherever possible. To allow the resection cavity to collapse as much as possible, the arachnoid space should be opened because, due to long-term pressure in slowly expanding tumors, it may be broadly adherent to the dura mater and completely obliterated.

In our treatment regimen for IMSCL, we adopted a routine MPS scheme based on the NASCIS II findings that investigated traumatic spinal cord injuries. ${ }^{37-39} \mathrm{In}$ 
NASCIS II, 24 hours of early MPS treatment $(<8$ hours after injury) showed an improvement in sensory function at the 6-month follow-up and in motor function at the 1-year follow-up. ${ }^{37,40,41} \mathrm{An}$ initial bolus of $30 \mathrm{mg} / \mathrm{kg}$ was applied before myelotomy, followed by a continuous application of $5.4 \mathrm{mg} / \mathrm{kg} / \mathrm{hr}$ for 24 hours. While there is criticism concerning the NASCIS II scheme, it may not apply to its use in resection of IMSCL. The controversy concerning the timing of MPS application is irrelevant to the elective surgical situation since it coincides with the timing of the trauma and exactly mimics the situation of an experimental spinal cord trauma in a laboratory situation, and therefore is different from an accident situation, where the exact mechanism of the trauma remains unclear. Also, the complications associated with high-dose steroids were found in a collective of patients who sustained severe trauma, often with significant comorbidities.

With the illustrated evolution of departmental standards, it appears that a wide spectrum of even formidable lesions can be safely approached. Establishing rigid schemes for this kind of surgery over the last 35 years has led to shortened hospital stays, shortened procedures, and the elimination of many obstacles inhibiting a smooth workflow. Key elements in this regard are optimal neuroimaging, monitoring, MPS, standardized laminotomy, transdural ultrasound, the availability of experienced neuropathology for frozen section, the awareness of pitfalls (e.g., trapped CSF, monitoring artifacts, unexpected histology), and the presence of a small, dedicated team to build a learning experience. The use of a $\mathrm{CO}_{2}$ laser may be departmentally specific, as others use different microsurgical technology.

\section{Conclusions}

Our series offers evidence that for rare lesions, which can have a broad variety of individual presentation, sufficient frequency of a procedure, and limitation to a small team with infrequent changes of personnel, allows for incremental refinements so that even a rare procedure can become standardized. This also ensures that optimal techniques will be passed on to trainees, maintaining a constant high quality for the outcome of the patients. Whatever these refinements are in specialized centers, the main emerging aspect is the elimination of a defeatist view in patients and referring physicians toward intramedullary lesions, to gain confidence from series like this that most patients will be able to walk home and continue with their normal lives, and even be cured in cases of low-grade endophytic tumors.

\section{Acknowledgments}

We thank Luca Papavero and Eric Fritzsche, who were a longterm part of the team, which began with Hans-Dietrich Herrmann and Loris Cristante. We also thank Tammam Abboud, Cindy Schwarz, and Romy See for intraoperative monitoring. We thank Sabine Wuttke for the excellent graphical support in this paper.

\section{References}

1. Boström A, Kanther NC, Grote A, Boström J. Management and outcome in adult intramedullary spinal cord tumours: a 20-year single institution experience. BMC Res Notes. 2014;7: 908.
2. Grimm S, Chamberlain MC. Adult primary spinal cord tumors. Expert Rev Neurother. 2009;9(10):1487-1495.

3. Hirano K, Imagama S, Sato K, et al. Primary spinal cord tumors: review of 678 surgically treated patients in Japan. A multicenter study. Eur Spine J. 2012;21(10):2019-2026.

4. Juthani RG, Bilsky MH, Vogelbaum MA. Current management and treatment modalities for intramedullary spinal cord tumors. Curr Treat Options Oncol. 2015;16(8):39.

5. Karikari IO, Nimjee SM, Hodges TR, et al. Impact of tumor histology on resectability and neurological outcome in primary intramedullary spinal cord tumors: a single-center experience with 102 patients. Neurosurgery. 2011;68(1):188-197.

6. Schellinger KA, Propp JM, Villano JL, McCarthy BJ. Descriptive epidemiology of primary spinal cord tumors. $J$ Neurooncol. 2008;87(2):173-179.

7. Tobin MK, Geraghty JR, Engelhard HH, et al. Intramedullary spinal cord tumors: a review of current and future treatment strategies. Neurosurg Focus. 2015;39(2):E14.

8. Samartzis D, Gillis CC, Shih P, et al. Intramedullary spinal cord tumors: part I-epidemiology, pathophysiology, and diagnosis. Global Spine J. 2015;5(5):425-435.

9. Kakareka M, Moncman R, Georges J, et al. Pediatric spinal cord biopsy: a case series from a high-volume referral center. J Clin Neurosci. 2019;65:34-40.

10. Richards $\mathrm{O}$, Goacher $\mathrm{E}, \mathrm{Pal} \mathrm{D}$, et al. Intramedullary spinal cord tumours - a single centre, 10-year review of clinical and pathological outcomes. Br J Neurosurg. 2021;35(2):125-128.

11. Samuel N, Tetreault L, Santaguida C, et al. Clinical and pathological outcomes after resection of intramedullary spinal cord tumors: a single-institution case series. Neurosurg Focus. 2016;41(2):E8.

12. Schebesch KM, Mueller S, Wendl C, et al. Recurrence rates and functional outcome after resection of intrinsic intramedullary tumors. Clin Neurol Neurosurg. 2015;134:60-66.

13. Seki T, Hida K, Yano S, et al. Surgical outcomes of highgrade spinal cord gliomas. Asian Spine J. 2015;9(6):935-941.

14. Svoboda N, Bradac O, de Lacy P, Benes V. Intramedullary ependymoma: long-term outcome after surgery. Acta Neurochir(Wien). 2018;160(3):439-447.

15. Cristante L, Hermann HD. Radical excision of intramedullary cavernous angiomas. Neurosurgery. 1998;43(3):424-431.

16. Cristante L, Herrmann HD. Surgical management of intramedullary hemangioblastoma of the spinal cord. Acta Neurochir(Wien). 1999;141(4):333-340.

17. Cristante L, Herrmann HD. Surgical management of intramedullary spinal cord tumors: functional outcome and sources of morbidity. Neurosurgery. 1994;35(1):69-76.

18. Cooper PR, Epstein F. Radical resection of intramedullary spinal cord tumors in adults. Recent experience in 29 patients. J Neurosurg. 1985;63(4):492-499.

19. Payer S, Mende KC, Westphal M, Eicker SO. Intramedullary spinal cord metastases: an increasingly common diagnosis. Neurosurg Focus. 2015;39(2):E15.

20. Eicker SO, Floeth FW, Kamp M, et al. The impact of fluorescence guidance on spinal intradural tumour surgery. Eur Spine J. 2013;22(6):1394-1401.

21. Westphal M. Intramedullary tumors. In: Tonn JC, Reardon DA, Rutka JT, Westphal M, eds. Oncology of CNS Tumors. 3rd ed. Springer; 2019:633-657.

22. Aghakhani N, Messerer M, David P, et al. Intramedullary ependymomas: a French retrospective multicenter study of 221 cases. Article in French. Neurochirurgie. 2017;63(5): 391-397.

23. Brotchi J. Intramedullary astrocytomas surgery in adult patients: the rationale for cautious surgery. World Neurosurg. 2013;80(5):e139-e140.

24. Brotchi J, Noterman J, Baleriaux D. Surgery of intramedullary spinal cord tumours. Acta Neurochir (Wien). 1992;116(24):176-178. 
25. Klekamp J. Treatment of intramedullary tumors: analysis of surgical morbidity and long-term results. J Neurosurg Spine. 2013;19(1):12-26.

26. Kucia EJ, Bambakidis NC, Chang SW, Spetzler RF. Surgical technique and outcomes in the treatment of spinal cord ependymomas, part 1: intramedullary ependymomas. Neurosurgery. 2011;68(1)(Suppl Operative):57-63.

27. Katsigiannis S, Carolus AE, Schmieder K, Brenke C. Posterolateral myelotomy for intramedullary spinal cord tumors: the other way to do it? Acta Neurochir (Wien). 2020;162(1): 101-107.

28. Sweeney KJ, Reynolds M, Farrell M, Bolger C. Gross total resection rates of grade II/III intramedullary ependymomas using the surgical strategy of en-bloc resection without intraoperative neurophysiological monitoring. Br J Neurosurg. 2017;31(3):364-368.

29. Jin SH, Chung CK, Kim CH, et al. Multimodal intraoperative monitoring during intramedullary spinal cord tumor surgery. Acta Neurochir (Wien). 2015;157(12):2149-2155.

30. Van Velthoven V, Reinacher PC, Klisch J, et al. Treatment of intramedullary hemangioblastomas, with special attention to von Hippel-Lindau disease. Neurosurgery. 2003;53(6): 1306-1314.

31. Mende KC, Krätzig T, Mohme M, et al. Keyhole approaches to intradural pathologies. Neurosurg Focus. 2017;43(2):E5.

32. Ahmed R, Menezes AH, Awe OO, et al. Long-term incidence and risk factors for development of spinal deformity following resection of pediatric intramedullary spinal cord tumors. J Neurosurg Pediatr. 2014;13(6):613-621.

33. Knafo S, Court C, Parker F. Predicting sagittal deformity after surgery for intramedullary tumors. J Neurosurg Spine. 2014;21(3):342-347.

34. McGirt MJ, Garcés-Ambrossi GL, Parker SL, et al. Shortterm progressive spinal deformity following laminoplasty versus laminectomy for resection of intradural spinal tumors: analysis of 238 patients. Neurosurgery. 2010;66(5):10051012.

35. Montano N, Trevisi G, Cioni B, et al. The role of laminoplasty in preventing spinal deformity in adult patients submitted to resection of an intradural spinal tumor. Case series and literature review. Clin Neurol Neurosurg. 2014;125:69-74.
36. Nakamura M, Tsuji O, Iwanami A, et al. Central neuropathic pain after surgical resection in patients with spinal intramedullary tumor. J Orthop Sci. 2012;17(4):352-357.

37. Bracken MB, Shepard MJ, Collins WF, et al. A randomized, controlled trial of methylprednisolone or naloxone in the treatment of acute spinal-cord injury. Results of the Second National Acute Spinal Cord Injury Study. $N$ Engl J Med. 1990;322(20):1405-1411.

38. Tsutsumi S, Ueta T, Shiba K, et al. Effects of the Second National Acute Spinal Cord Injury Study of high-dose methylprednisolone therapy on acute cervical spinal cord injuryresults in spinal injuries center. Spine (Phila Pa 1976). 2006; 31(26):2992-2997.

39. Young W. NASCIS. National Acute Spinal Cord Injury Study. J Neurotrauma. 1990;7(3):113-114.

40. Bracken MB. Steroids for acute spinal cord injury. Cochrane Database Syst Rev. 2012;1(1):CD001046.

41. Bracken MB, Shepard MJ, Collins WF Jr, et al. Methylprednisolone or naloxone treatment after acute spinal cord injury: 1-year follow-up data. Results of the second National Acute Spinal Cord Injury Study. J Neurosurg. 1992;76(1):23-31.

\section{Disclosures}

Dr. Eicker: honoraria from Stryker and Spineart.

\section{Author Contributions}

Conception and design: Westphal, Eicker. Acquisition of data: Mende, Eicker. Analysis and interpretation of data: Mende. Drafting the article: Westphal, Eicker. Critically revising the article: Westphal. Approved the final version of the manuscript on behalf of all authors: Westphal. Statistical analysis: Mende.

\section{Correspondence}

Manfred Westphal: University Medical Center Hamburg-Eppendorf, Hamburg, Germany. westphal@uke.de. 